\title{
Etude de la stabilité de l'iode dans le sel iodé
}

\author{
Mahamat SEID ALI ${ }^{1 *}$, Abdelsalam TIDJANI ${ }^{1}$ et Nicolas Cyrille AYESSOU ${ }^{2}$ \\ ${ }^{1}$ Université de N'Djaména, B.P.1117, N'Djaména, Tchad. \\ ${ }^{2}$ Ecole Supérieure Polytechnique, UCAD, Dakar BP 5085, Sénégal. \\ *Auteur Correspondant ; E-mail : seidaligarga@gmail.com
}

\section{REMERCIEMENTS}

Les travaux ont bénéficié du soutien financier de la Commission Nationale pour la Formation de Formateur (CONFOFOR) et technique du Centre National de Recherche pour le Développement (CNRD). Nous tenons à exprimer toute notre reconnaissance aux promoteurs de ce projet ainsi qu'au Ministère de l'Enseignement Supérieur et de la Recherche Scientifique du Tchad.

\section{RESUME}

Le sel iodé est un sel de table mélangé avec une faible quantité de sel d'iode, de façon à diminuer les risques de déficiences en iode dans l'organisme, déficiences qui sont à l'origine de problèmes au niveau de la glande thyroïdienne. Près de 800 millions de personnes dans le monde sont affectées par la carence en iode et sa conséquence la plus visible, le goitre endémique. Cette situation concernerait $20 \%$ de la population du globe, ce qui constitue un vrai problème de santé publique. L'objectif de ces travaux scientifiques consiste à étudier la stabilité de l'iode par rapport à la nature du sel et l'emballage utilisé pour mieux appréhender les sources de déperdition pendant la durée de stockage du sel iodé ainsi que le comportement de l'iode lors de la cuisson. L'influence de la nature du sel a été étudiée par le suivi de l'iode pendant trois mois à intervalle d'une semaine sur trois types de sel. Le suivi de l'impact de l'emballage utilisé sur la déperdition d'iode a été effectué sur quatre types d'emballage durant la même période. Les effets de la température et de la durée d'exposition ont également été étudiés pendant la cuisson. Au terme des résultats de cette étude, il ressort que la déperdition d'iode dans le sel est liée à la granulométrie des cristaux et à la présence des matières insolubles. Par rapport à l'impact de l'emballage utilisé, l'iode est quasiment resté stable dans le sel.

(c) 2015 International Formulae Group. All rights reserved.

Mots clés : Stabilité, cuisson, sel iodé, emballage, granulométrie, déperdition.

\section{Study of the stability of iodine in iodized salt}

\begin{abstract}
Iodized salt is table salt mixed with a small amount of iodine salt in order to reduce the risk of iodine deficiency in the body, deficiencies that are causing problems at the thyroid gland. Nearly 800 million people worldwide are affected by iodine deficiency and its most visible result, endemic goitre. This would cover $20 \%$ of the world population, which is a real public health problem. The purpose of this scientific work is to study the stability of iodine in relation to the nature of the salt and the packaging used to better understand the sources of loss during the period of storage of iodized salt and the behavior of the iodine during cooking. The
\end{abstract}


influence of the nature of the salt was investigated by monitoring the iodine for three months at weekly intervals on three types of salt. Monitoring the impact of packaging used on the iodine loss was performed on four types of packaging in the same period. The effect of temperature and duration of exposure have also been studied during cooking. After the results of this study, it appears that the loss of iodine in the salt is related to the particle size of the crystals and to the presence of insolubles. With respect to the impact of the packaging used, the iodine remained almost stable

() 2015 International Formulae Group. All rights reserved.

Keywords: stability, cooking, iodized salt, packing, granulometry, loss.

\section{INTRODUCTION}

La carence en micronutriments mine en silence la santé de l'homme. Ils agissent en petite dose et ne sont pas fabriqués par l'organisme ou le sont faiblement fabriqués (Yomadji-Outengar, 2002). Les sels minéraux et les oligoéléments sont indispensables à notre santé, car ils assurent de nombreuses fonctions vitales dans notre organisme. Cependant, leurs apports alimentaires sont de plus en plus moindres et leurs carences constituent aujourd'hui un problème majeur de santé publique à travers le monde entier, particulièrement dans les pays pauvres ou en voie de développement (Ministère de la Santé, 2001).

Parmi les carences en micronutriments, figure la carence en iode qui constitue l'une des carences les plus répandues dans le monde et touche pratiquement toutes les tranches d'âge; les enfants et les femmes restent les plus concernés par ce fléau surtout en Afrique et en Asie du Sud-Est (FAO, 2015).

Elle a des conséquences graves sur la santé (goitre, crétinisme, retard mental, etc.) qui affectent aussi le développement socioéconomique (Allen, 2011). L'iode est un oligo élément essentiel pour la vie (Phyllis et Lyday, 2005). En effet, lorsque les normes concernant la quantité d'iode nécessaire au bon fonctionnement de l'organisme ne sont pas atteintes $(150 \mu \mathrm{g} / \mathrm{jour})$, il arrive que la thyroïde ne soit plus en mesure de synthétiser l'hormone thyroïdienne en quantité suffisante. La faible concentration sanguine qui en résulte est la cause principale d'un certain nombre d'anomalies du métabolisme et du développement, plus connues sous le terme de
Troubles Dus à la Carence en Iode (TDCI) forgés en 1983 par Basil Hetzel pour désigner le large éventail des effets négatifs de la carence en iode (Ministère de la Santé,2008).

L'adoption de ce terme a marqué un tournant en sensibilisant au problème et en incitant les gouvernements et les organisations internationales à agir (OMS, 1998). C'est ainsi que l'iodation universelle du sel comme stratégie de prévention et de contrôle des TDCI a été recommandée par l'OMS et le nombre de pays ayant une carence en iode a diminué dans les années 2007 (de Benoist, 2002 ; Anderson, 2010). Toutefois, la stabilité de l'agent iodant utilisé dans le sel a toujours constitué une entrave même si certaines solutions ont été adoptées comme l'utilisation de l'iodate de potassium, jugé plus stable, à la place de l'iodure de potassium (Lafoix, 2005). Le but de ce travail est de contribuer à trouver des solutions à ce problème qui est un facteur limitant la disponibilité de l'iode. En même temps, l'accent sera mis sur tout paramètre pouvant augmenter la biodisponibilité de l'iode que le sel iodé est censé apporter afin de permettre aux populations de se préserver des troubles que peuvent causer les TDCI.

\section{MATERIEL ET METHODES}

Le Sénégal est un pays de forte production de sel et se trouve au rang des grands producteurs en Afrique de l'Ouest. Pour une production annuelle estimée environ à 500000 tonnes, le Sénégal assure les 350000 tonnes suivi du Ghana, environ 100000 tonnes (Lagnane, 2012). Afin d'étudier les paramètres qui peuvent 
influencer sur la stabilité de l'iode dans le sel iodé, nous avons procédé tout d'abord à une caractérisation chimique de sels bruts produits et échantillonnés dans les différents sites de production (artisanale et industrielle) de sel iodé au Sénégal. Pour mieux appréhender les sources de déperdition d'iode que le sel iodé est censé apporter, nous avons ensuite procédé à une étude du comportement de l'iode lors de la cuisson.

\section{Caractérisation chimique de sels bruts produits au Sénégal \\ Echantillonnage de sels bruts}

Au Sénégal, le sel iodé est produit industriellement (par SNSS et SELSINE) mais aussi artisanalement (par des producteurs organisés en GIE à travers tout le pays). Nous avons visité quelques sites de production de sel iodé. Le but de cette visite était de comprendre les techniques d'extraction et d'effectuer un échantillonnage pour la caractérisation des sels bruts produits dans ces différents sites. A l'issu de cette visite, nous avons échantillonné au total 12 types de sels bruts comme indiqué dans le Tableau1.

\section{Détermination des différents paramètres}

Le Codex Alimentarius définit le sel de qualité alimentaire comme étant un produit cristallin se composant principalement de chlorure de sodium $(\mathrm{NaCl})$. Il peut provenir de la mer, de gisements souterrains de sel de gemme, ou encore de saumure naturelle. Selon la norme sénégalaise «NS 03-0017 qui traite des Additifs alimentaires- sel de cuisine », le sel, utilisé en tant qu'ingrédient alimentaire destiné aussi bien à la vente directe aux consommateurs qu'à l'industrie alimentaire, doit respecter certaines exigences de qualité. Les paramètres essentiels sont la teneur en chlorure de sodium, l'humidité, les matières insolubles et les contaminants naturels comme le calcium.

\section{Teneur en chlorure de sodium}

La méthode E 534-98 de l'ASTM (American Society for Testing and Materials) a été utilisée pour le dosage du chlorure de sodium.

\section{Humidité}

La méthode utilisée est celle de la norme française NF V04 - 401 d'Avril 2001.

\section{Matières insolubles}

La méthode utilisée est celle de la norme ISO 2479 - 1972.

\section{Oxydabilité}

Elle correspond à la quantité de matières organiques présentes dans le sel. C'est un paramètre qui n'est pas exigé par la norme. Elle a été introduite pour apprécier la proportion des matières organiques dans le sel. En effet, elles constituent une cause principale de déperdition d'iode dans le sel. La méthode de la détermination de l'oxydabilité au permanganate de potassium des eaux (Rodier, 1984) a été utilisée.

\section{Dosage du calcium}

Il est effectué par spectrophotométrie d'absorption atomique avec flamme. La méthode utilisée est celle du dosage du calcium dans les eaux naturelles (Rodier, 1996).

\section{Etude de la stabilité de l'iode dans le sel iodé}

L'étude de la stabilité de l'iode est importante pour deux raisons. D'une part, il est indispensable que le sel iodé renferme la teneur en iode réelle préconisée par les autorités médicales afin de prévenir les TDCI. D'autre part, les producteurs doivent être assurés que leurs produits satisferont aux règles édictées par le gouvernement au sujet du taux d'iodation.

La stabilité de l'iode dans le sel dépend de la teneur en eau, de l'acidité et de la pureté du sel auquel l'iode est ajouté. Pour réduire les pertes d'iode pendant le stockage, le sel iodé doit être aussi pur et sec que possible et 
doit être correctement emballé (Allen et al., 2011).

Pour cette étude, l'accent a été mis sur les paramètres suivants :

la nature du sel: pour ce paramètre, la stabilité de l'iode par rapport à la granulométrie et la pureté du sel a été évaluée.

l'emballage : il s'agit de proposer un nouveau type d'emballage au cas où celui utilisé constituerait un facteur de déperdition d'iode.

\section{Influence de la nature du sel sur la stabilité de l'iode}

La méthodologie consiste à faire un suivi de l'iode sur trois types de sels iodés dont les caractéristiques sont connues comme le montre le Tableau 2. Les trois types de sels sont: le sel fin, le sel criblé et le sel tout venant. Le sel tout venant est du sel n'ayant subi aucun traitement (lavage, raffinage, broyage, etc.) avec une granulométrie diverse. Le sel criblé est formé de cristaux de taille différente, obtenu par tamisage après broyage lors de la production de sel fin.

Le suivi a été réalisé pendant 3 mois (12 semaines) par intervalle d'une semaine. Pour chaque type, le sel est emballé dans des sachets de 25 grammes de PP (polypropylène). Les échantillons de sel sont mis sur le marché (à Dakar) dans les mêmes conditions que ceux destinés à la vente. Chaque semaine, le dosage de l'iode a été réalisé sur trois échantillons de chaque type de sel et l'analyse a été effectuée en double (soit au total 18 dosages d'iode par semaine).

\section{Impact de l'emballage utilisé sur la déperdition d'iode}

Le suivi est réalisé aussi sur 3 mois. Quatre types d'emballages ont été utilisés : en plus du PEHD (polyéthylène haute densité) et du PP (polypropylène) recommandés pour le sel iodé, nous avons utilisé le TRIPLEX et le DUPLEX. Le TRIPLEX est utilisé souvent pour le lait en poudre, il est constitué d'une couche de PET (polyéthylène), de PEHD et d'un film métallisé. Le DUPLEX est utilisé pour l'eau de javel, c'est du PET et du PEHD renforcé de noir. Les caractéristiques de ces différents emballages sont présentées dans le Tableau 3.

Comme représenté dans la Figure 1, il est réparti dans des sachets de 25 grammes pour chaque type d'emballage. Trois endroits ont été ciblés pour cette étude : un marché à Dakar, un marché à Matam et le laboratoire dans des conditions maîtrisées de température et de luminosité. Le dosage de l'iode est réalisé chaque semaine pour le suivi à Dakar et au laboratoire et chaque mois pour Matam. Il est effectué en double sur trois échantillons de chaque type d'emballage (soit 48 dosages d'iode par semaine pour les suivis de Dakar et laboratoire et 24 par mois pour Matam).

\section{Etude du comportement de l'iode lors de la cuisson}

Pour plus d'illustrations sur la stabilité de l'iode dans le sel, le comportement de l'iode lors de la cuisson a été étudié. En effet, même si la teneur en iode du sel utilisé pour la prophylaxie contre les TDCI est adéquate et que lors de la cuisson cet iode est détruit; il n'y aura pas d'apport en iode et l'objectif n'est pas atteint. Ainsi, il serait intéressant de mettre l'accent sur tout ce qui peut augmenter la biodisponibilité de l'iode afin de permettre aux populations de bien asseoir leur santé. La méthodologie consiste à étudier deux facteurs qui peuvent influencer sur l'iode lors de la cuisson : la température et la durée de cuisson. La température est variée de 70 à $100{ }^{\circ} \mathrm{C}$ par intervalle de $5{ }^{\circ} \mathrm{C}$ pour $10 \mathrm{~min}, 30 \mathrm{~min}, 1 \mathrm{H}, 1$ $\mathrm{H} 30$ et $2 \mathrm{H}$. Dans un premier temps, le bain marie a été réglé à $70^{\circ} \mathrm{C}$ et cinq erlenmeyers contenant chacun $10 \mathrm{~g}$ de sel iodé (dont la teneur initiale en iode est connue 116,06 ppm) dissous dans $50 \mathrm{ml}$ d'eau distillée y ont été introduits. Un erlenmeyer a été retiré au bout de $10 \mathrm{~min}$, de $30 \mathrm{~min}$, de $1 \mathrm{H}$, de $1 \mathrm{H} 30$ et de $2 \mathrm{H}$. Le même principe est appliqué à $75^{\circ} \mathrm{C}$, $80{ }^{\circ} \mathrm{C}, 85^{\circ} \mathrm{C}, 90^{\circ} \mathrm{C}, 95^{\circ} \mathrm{C}$ et à $100{ }^{\circ} \mathrm{C}$. Ensuite, l'iode a été dosé dans chaque erlenmeyer par la méthode de dosage de l'iode dans le sel d'AOAC. 


\section{Dosage de l'iode dans le sel}

La méthode de dosage de l'iode utilisée est celle de l' "Association of official Analytical Chemists (AOAC)» (AOAC, 1974). Par exemple, Dix grammes de sel iodé ont été introduits dans un erlenneyer de 250 ; $50 \mathrm{ml}$ d'eau distillée y ont été ajoutées. Apres dissolution totale par agitation, $1 \mathrm{ml} \mathrm{de} \mathrm{H}_{2} \mathrm{SO}_{4}$ $(1 \mathrm{M})$ et $5 \mathrm{ml}$ de $\mathrm{KI}$ ont été ajoutés. Après une incubation de 10 min à l'obscurité, la solution a été dosée par du $\mathrm{Na}_{2} \mathrm{~S}_{2} \mathrm{O}_{3}(2,5 \mathrm{mmol} / \mathrm{l})$ jusqu'à disparition de la coloration jaune. Pour la vérification, si c'est le cas d'une coloration violette, l'amidon a été ajouté dans la solution.

\section{Expression des résultats}

L'expérience montre que $1 \mathrm{ml}$ de $\mathrm{Na}_{2} \mathrm{~S}_{2} \mathrm{O}_{3}(2,5 \mathrm{mmol} / \mathrm{l})$ réduit $0,1058 \mathrm{mg}$ d'iode. En multipliant par le volume de $\mathrm{Na}_{2} \mathrm{~S}_{2} \mathrm{O}_{3}$ utilisé pour réduire l'iode contenu dans $10 \mathrm{~g}$, on a la quantité d'iode en $\mathrm{mg}$ dans $10 \mathrm{~g}$ de sel. En multipliant par 100, on a le résultat en $\mathrm{mg}$ d'iode contenu dans $1000 \mathrm{~g}$, ce qui correspond à $1000 \mathrm{mg}$ d'iode par kg de sel c'est-à-dire ppm.

Iode $(\mathrm{ppm})=$ Volume $\mathrm{Na}_{2} \mathrm{~S}_{2} \mathrm{O}_{3} \times 0,1058 \times 100$

\section{RESULTATS}

Les échantillons de sels analysés sont pratiquement secs mais $33 \%$ contiennent des taux d'impuretés ou d'insolubilité supérieurs à $0,3 \%$. Cependant, $16,6 \%$ des échantillons présentent des taux de $\mathrm{NaCl}$ inférieurs à $95 \%$ dont les teneurs ne sont pas trop différentes de la référence. L'oxydabilité étant liée aux impuretés particulièrement aux matières organiques, elle n'est jamais nulle dans tous les échantillons analysés. En plus, 100\% des échantillons de sels bruts contiennent du calcium sous forme de traces.

Pour le Lac Rose, les caractéristiques de l'échantillon numéro 1 sont très différentes de celles du numéro 2 . Ce dernier est moins pur $(91,85 \%)$ avec des taux d'humidité $(7,05$ $\%)$ et d'impuretés $(4,66 \%)$ élevés et hors normes. Par rapport à la nature du sel, l'étude a donné des résultats qui sont consignés dans le Tableau 5 : il s'agit des valeurs moyennes de 6 dosages effectués, du taux de diminution en iode ( $\Delta$ iode) ainsi que les pourcentages de déperdition obtenus au cours des 12 semaines de suivi avec les trois types de sel.

Le Tableau 5 montre que la variation de la teneur en iode est plus importante pour le sel criblé et le sel tout venant que pour le sel fin. Elle est pratiquement constante pour le sel fin comparé aux autres types de sel.

Pour mieux illustrer cela, la Figure 2 donne les pourcentages de déperdition d'iode par rapport à la valeur initiale au cours des 12 semaines de suivi.

L'étude de la stabilité de l'iode par rapport à l'emballage utilisé a donné des résultats consignés dans les Tableaux $6 ; 7$ et 8 : il s'agit des valeurs moyennes de 6 dosages effectués, du taux de diminution en iode $(\Delta$ iode) ainsi que les pourcentages de déperdition obtenus au cours des 12 semaines de suivi avec les emballages pour les différents endroits de stockage.

Le Tableau 6 montre qu'au bout de 3 mois, la variation du taux d'iode par rapport à la teneur initiale $(140,85 \mathrm{ppm})$ n'est pas aussi importante et elle passe de 0,00 à $6,48 \mathrm{ppm}$ maximum. Aussi, les tendances sont les mêmes pour tous les types d'emballages utilisés pour cette expérimentation.

La Figure 4 donne les pourcentages de déperdition d'iode pour chaque type d'emballage au bout des 12 semaines de suivi.

Au bout des 12 semaines de suivi avec les emballages au Marché-Dakar, le pourcentage de déperdition d'iode est le même pour tous les emballages (TRIPLEX, DUPLEX, PEHD et PP) et varie entre 4,10 et $4,60 \%$.

Le Tableau 7 montre qu'au bout de 3 mois, la variation du taux d'iode par rapport à la teneur initiale $(140,85 \mathrm{ppm})$ n'est pas aussi importante et elle passe de 0,00 à 6,84 ppm maximum. Aussi, les tendances sont les 
mêmes pour tous les types d'emballages utilisés pour cette expérimentation.

La Figure 5 donne les pourcentages de déperdition d'iode pour chaque type d'emballage au bout des 12 semaines de suivi.

$\mathrm{Au}$ bout des 12 semaines de suivi avec les emballages au Laboratoire, le pourcentage de déperdition d'iode est le même pour tous les emballages (TRIPLEX, DUPLEX, PEHD et PP) et varie entre 3,85 et $4,85 \%$

Le Tableau 8 montre qu'au bout de 3 mois, la variation du taux d'iode par rapport à la teneur initiale (140,85 ppm) n'est pas aussi importante et elle passe de 0,00 à 6,66 ppm maximum. Aussi, les tendances sont les mêmes pour tous les types d'emballages utilisés pour cette expérimentation.
La Figure 6 donne les pourcentages de déperdition d'iode pour chaque type d'emballage au bout des 12 semaines de suivi.

$\mathrm{Au}$ bout des 12 semaines de suivi avec les emballages au Laboratoire, le pourcentage de déperdition d'iode est le même pour tous les emballages (TRIPLEX, DUPLEX, PEHD et PP) et varie entre 3,98 et $4,73 \%$.

Durant les 3 mois de suivi avec les différents lieux de stockage (Dakar, Matam et au Laboratoire), l'iode est quasiment resté stable dans le sel. Les taux de diminution en iode ( $\Delta$ iode), par rapport à la teneur initiale, obtenus au dernier dosage pour les différentes expérimentations est environ de 6,60 ppm correspondant à une déperdition de $4,68 \%$ en moyenne.

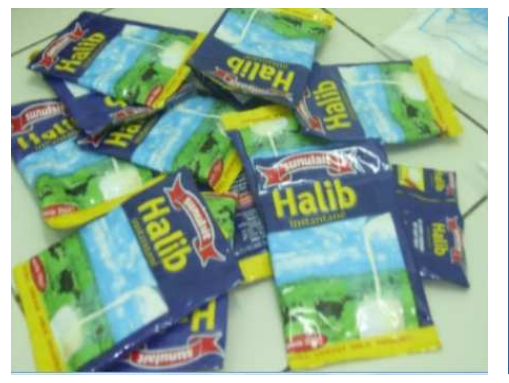

Emballage avec TRIPLEX

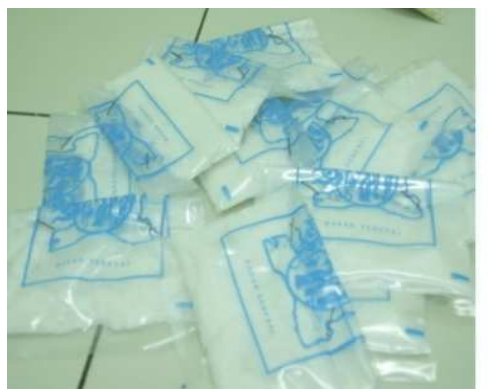

Emballage avec PEHD

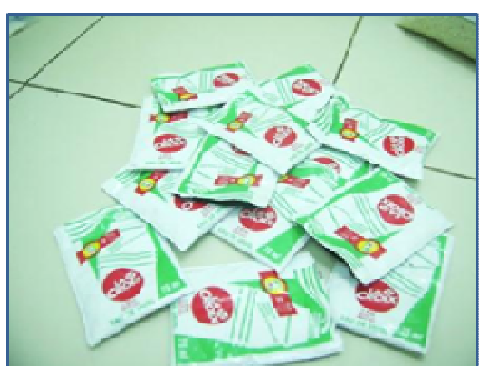

Emballage avec DUPLEX

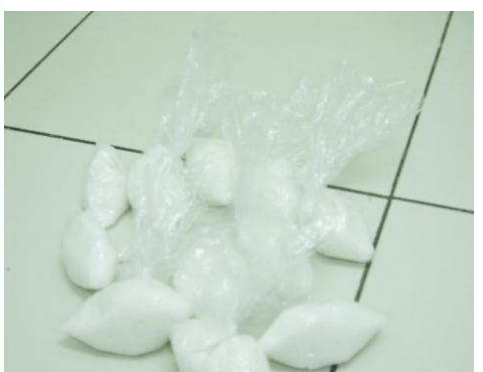

Emballage avec PP

Figure 1 : Types d'emballage. 
M. SEID ALI et al. / Int. J. Biol. Chem. Sci. 9(5): 2719-2734, 2015

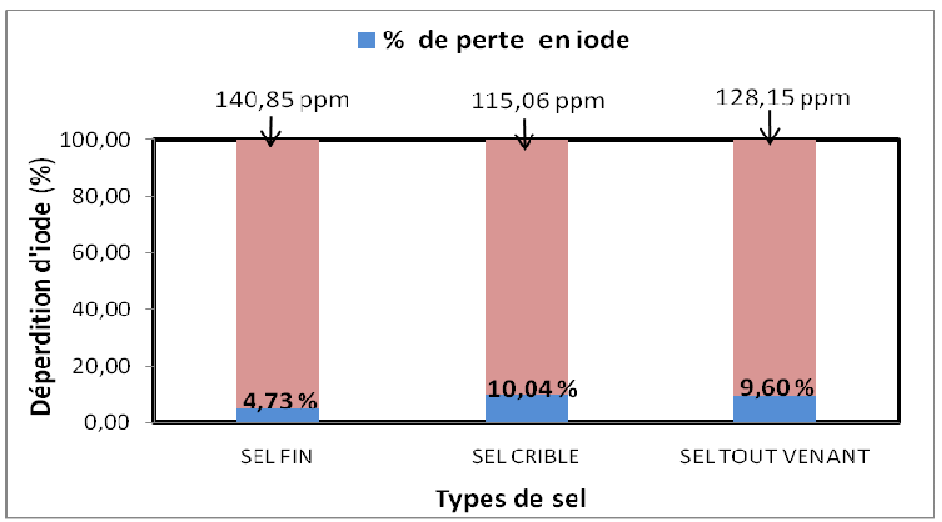

Figure 2: Déperdition d'iode au bout des 12 semaines de suivi avec les types de sel.

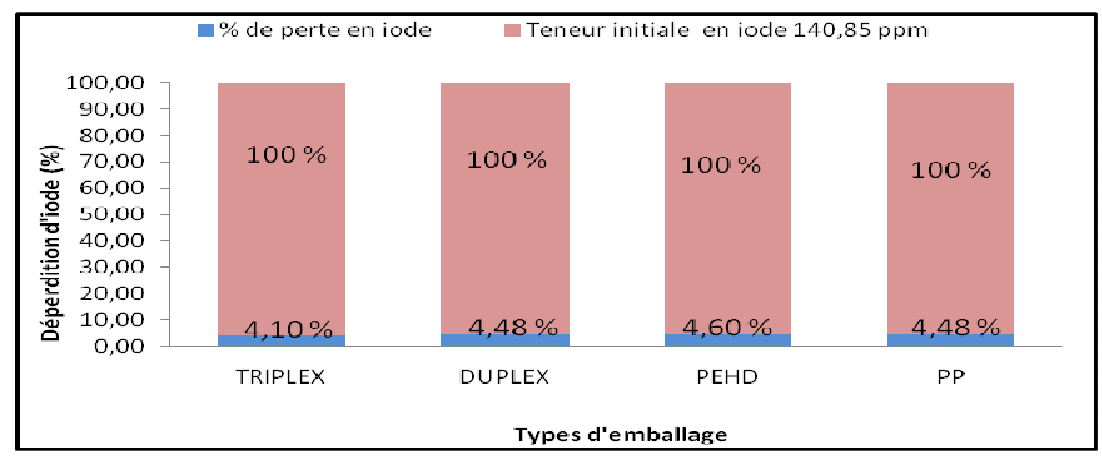

Figure 4 : Déperdition d'iode au bout des 12 semaines de suivi avec les emballages au MarchéDakar.

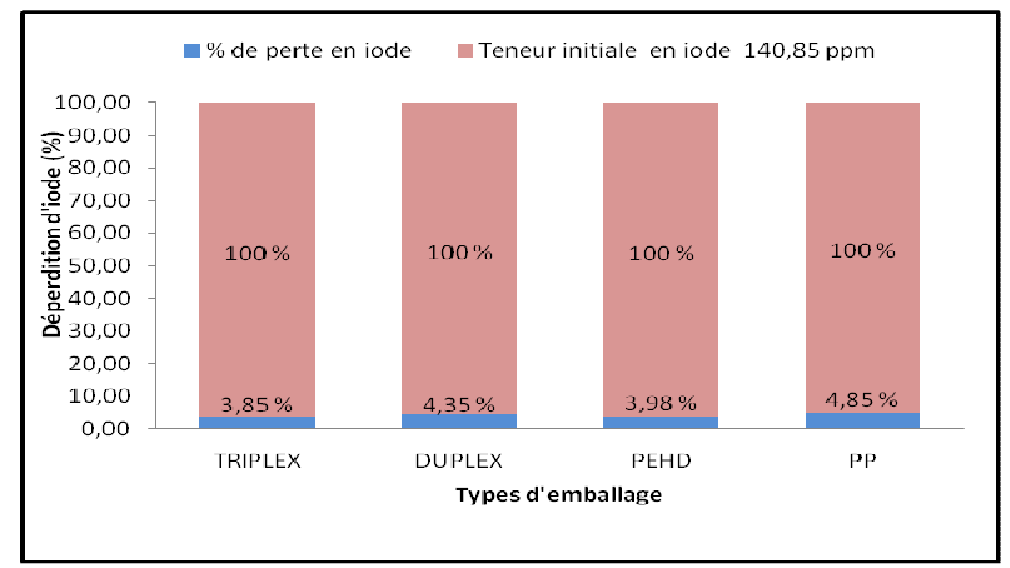

Figure 5: Déperdition d'iode au bout des 12 semaines de suivi avec les emballages au Laboratoire. 


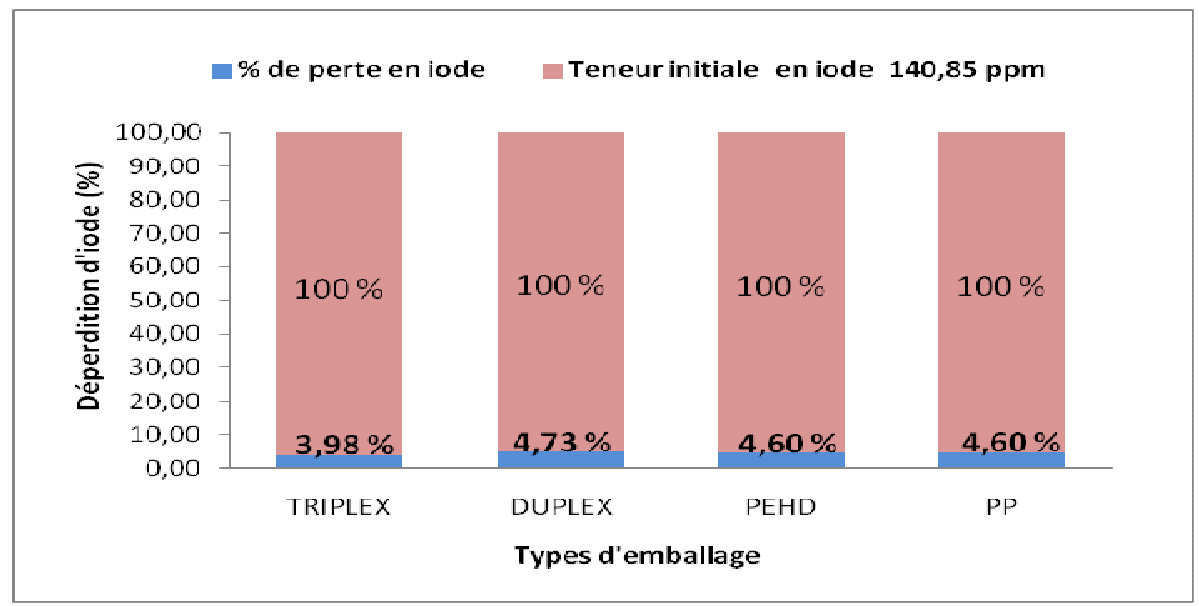

Figure 6 : Déperdition d'iode au bout des 3 mois de suivi avec les emballages au Marché - Matam.

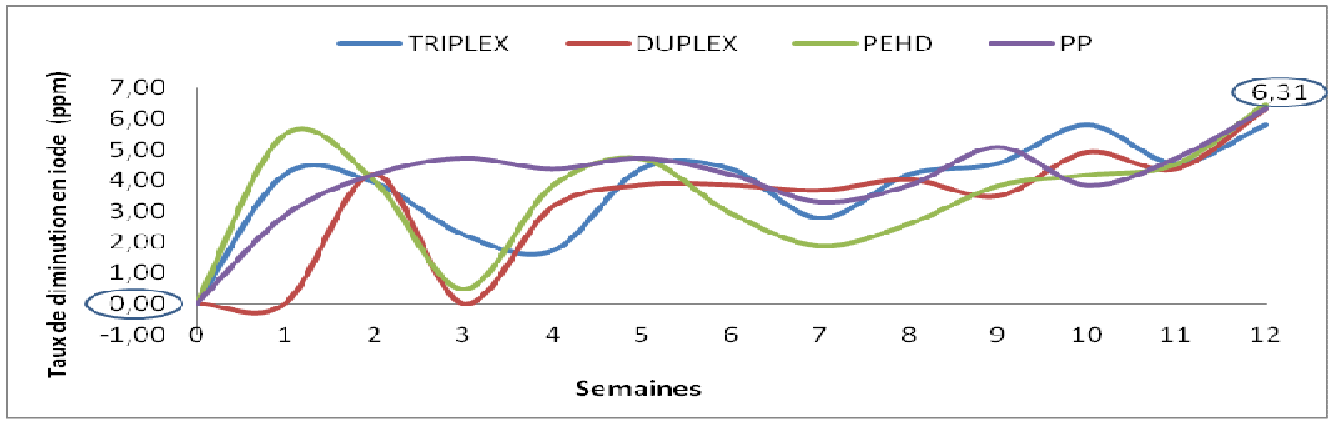

Figure 7: Variation du taux de diminution en iode durant le suivi avec les emballages au MarchéDakar.

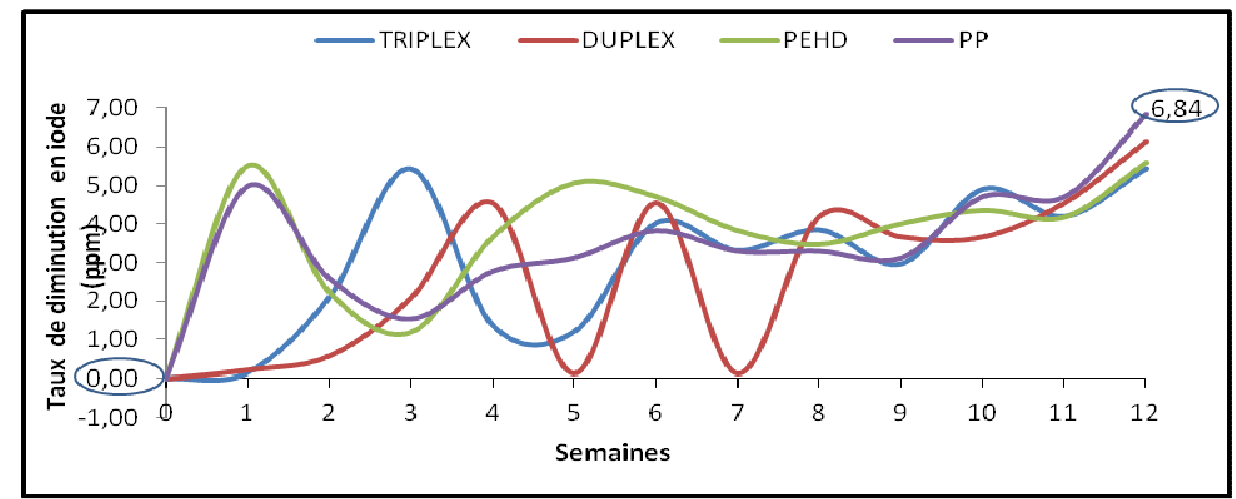

Figure 8: Variation du taux de diminution en iode durant le suivi avec les emballages au Laboratoire. 


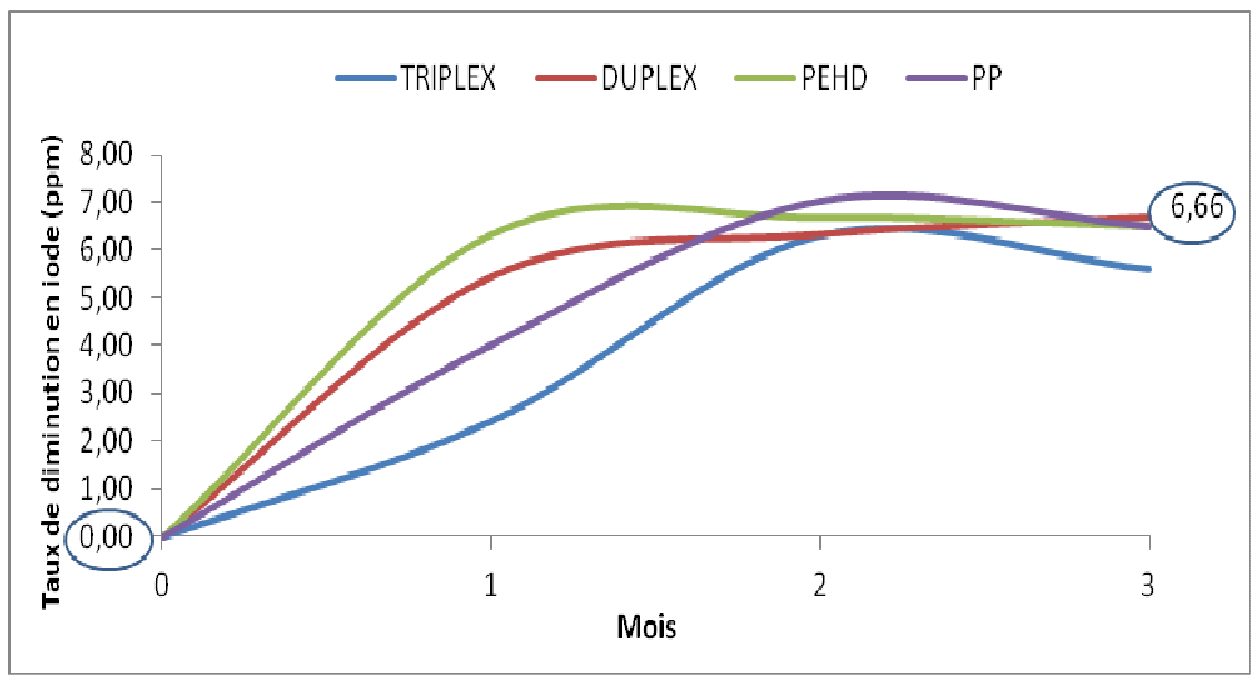

Figure 9: Variation du taux de diminution en iode durant le suivi avec les emballages au Marché Matam.

Tableau 1 : Liste des échantillons de sels bruts obtenus sur les sites de production.

\begin{tabular}{lcc}
\hline Régions & Sites & Nombres d'échantillons \\
\hline \multirow{3}{*}{ Kaolack } & SNSS & 2 \\
& Kaolack & 2 \\
& MakaKahone & 2 \\
\hline Fatick & Fatick & 2 \\
Dakar & Lac Rose & 2 \\
Saint-Louis & Gandiol & 2 \\
\hline Total & & 12 \\
\hline
\end{tabular}

Tableau 2 : Caractéristiques des types de sels utilisés pour le suivi.

\begin{tabular}{lccc}
\hline $\begin{array}{l}\text { Types de sel / } \\
\text { Paramètres }\end{array}$ & Sel fin & Sel criblé & Sel tout venant \\
\hline Teneur en $\mathrm{NaCl}(\%)$ & 100,00 & 100,00 & 100,00 \\
Humidité $(\%)$ & 2,38 & 0,14 & 3,11 \\
Matières insolubles $(\%)$ & 0,03 & 0,01 & 0,08 \\
Oxydabilité (mg/g) & 0,05 & 0,01 & 0,03 \\
Calcium $(\%)$ & $0,65.10^{-4}$ & $0,93.10^{-4}$ & $0,79.10^{-4}$ \\
Teneur en iode (ppm) & 140,85 & 115,06 & 128,15 \\
\hline
\end{tabular}


Tableau 3 : Caractéristiques des types de sel utilisés pour le suivi

\begin{tabular}{lcccc}
\hline $\begin{array}{l}\text { Emballages / } \\
\text { Paramètres }\end{array}$ & TRIPLEX & DUPLEX & PEHD & PP \\
\hline Epaisseur $(\mu)$ & 75 & 100 & $120-130$ & 70 \\
Opacité & 2,4 & 2,2 & 0,45 & 0 \\
\hline
\end{tabular}

Tableau 4 : Caractéristiques chimiques de sels bruts produits au Sénégal.

\begin{tabular}{|c|c|c|c|c|c|c|}
\hline \multicolumn{2}{|l|}{ Paramètres } & \multirow{2}{*}{$\begin{array}{c}\text { Teneur en } \mathrm{NaCl} \\
(\%)\end{array}$} & \multirow{2}{*}{$\begin{array}{c}\text { Humidité } \\
(\%)\end{array}$} & \multirow{2}{*}{$\begin{array}{c}\text { Matières } \\
\text { insolubles }(\%)\end{array}$} & \multirow{2}{*}{$\begin{array}{c}\begin{array}{c}\text { Oxydabilité } \\
(\mathrm{mg} / \mathrm{g})\end{array} \\
-\end{array}$} & \multirow{2}{*}{$\begin{array}{c}\text { Calcium (\%) } \\
0,1-0,40\end{array}$} \\
\hline Normes NS 03 & 0017 & & & & & \\
\hline & 1 & 100,00 & 2,38 & 0,03 & 0,05 & $0,65 \cdot 10^{-4}$ \\
\hline \multirow[t]{2}{*}{ SNSSS } & 2 & 100,00 & 0,14 & 0,01 & 0,01 & $0,93 \cdot 10^{-4}$ \\
\hline & 1 & 100,00 & 1,62 & 0,06 & 0,04 & $0,62 \cdot 10^{-4}$ \\
\hline \multirow[t]{2}{*}{ Kaolack } & 2 & 100,00 & 2,90 & 0,05 & 0,02 & $0,92 \cdot 10^{-4}$ \\
\hline & 1 & 100,00 & 1,89 & 0,07 & 0,04 & $0,73 \cdot 10^{-4}$ \\
\hline \multirow[t]{2}{*}{ Maka Kahone } & 2 & 99,45 & 1,30 & 0,11 & 0,05 & $10^{-4}$ \\
\hline & 1 & 100,00 & 4,37 & $0,36^{*}$ & 0,05 & $10^{-4}$ \\
\hline \multirow[t]{2}{*}{ Fatick } & 2 & 100,00 & 1,40 & $0,32^{*}$ & 0,07 & $1,20 \cdot 10^{-4}$ \\
\hline & 1 & 100,00 & 3,74 & 0,28 & 0,04 & $0,71 \cdot 10^{-4}$ \\
\hline \multirow[t]{2}{*}{ Lac Rose } & 2 & $91,85^{*}$ & $7,05^{*}$ & $4,66^{*}$ & 0,07 & $0,83 \cdot 10^{-4}$ \\
\hline & 1 & 95,06 & 2,79 & $1,14^{*}$ & 0,01 & $0,85 \cdot 10^{-4}$ \\
\hline Gandiol & 2 & $93,60 *$ & 3,88 & $0,64 *$ & 0,03 & $0,88 \cdot 10^{-4}$ \\
\hline
\end{tabular}


M. SEID ALI et al. / Int. J. Biol. Chem. Sci. 9(5): 2719-2734, 2015

Tableau 5 : Suivi du taux d'iode en fonction de la nature du sel.

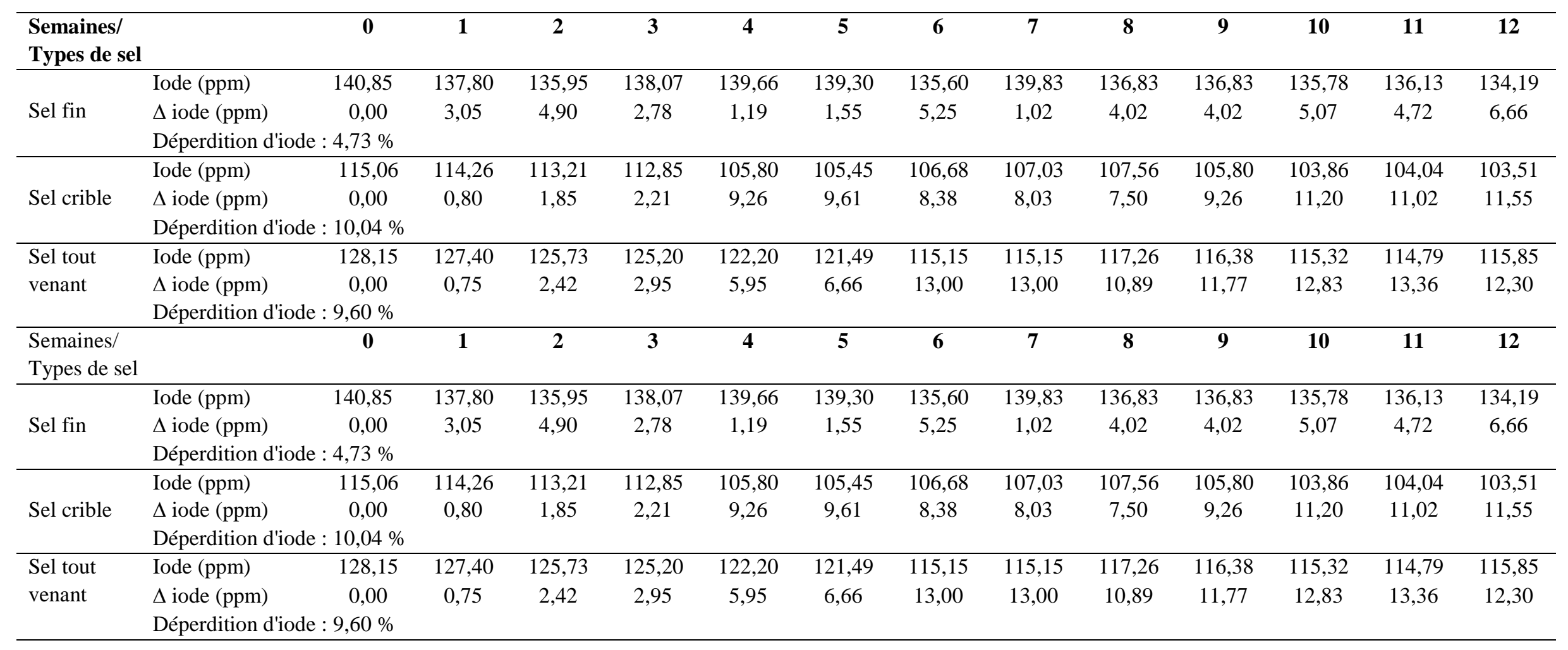


M. SEID ALI et al. / Int. J. Biol. Chem. Sci. 9(5): 2719-2734, 2015

Tableau 6 : Suivi du taux d'iode en fonction de l'emballage au Marché -Dakar.

\begin{tabular}{|c|c|c|c|c|c|c|c|c|c|c|c|c|c|c|}
\hline $\begin{array}{l}\text { Semaines/ } \\
\text { Emballages }\end{array}$ & & $\mathbf{0}$ & 1 & 2 & 3 & 4 & 5 & 6 & 7 & 8 & 9 & 10 & 11 & 12 \\
\hline \multirow{3}{*}{ TRIPLEX } & Iode (ppm) & 140,85 & 136,66 & 136,92 & 138,60 & 139,13 & 136,48 & 136,48 & 138,07 & 136,66 & 136,31 & 135,07 & 136,31 & 135,07 \\
\hline & $\Delta$ iode $(\mathrm{ppm})$ & 0,00 & 4,19 & 3,93 & 2,25 & 1,72 & 4,37 & 4,37 & 2,78 & 4,19 & 4,54 & 5,78 & 4,54 & 5,78 \\
\hline & Déperdition d & $4,10 \%$ & & & & & & & & & & & & \\
\hline \multirow{3}{*}{ DUPLEX } & Iode (ppm) & 140,85 & 141,24 & 136,66 & 141,60 & 137,72 & 137,01 & 137,01 & 137,19 & 136,83 & 137,36 & 135,95 & 136,48 & 134,54 \\
\hline & $\Delta$ iode $(\mathrm{ppm})$ & 0,00 & 0,00 & 4,19 & 0,00 & 3,13 & 3,84 & 3,84 & 3,66 & 4,02 & 3,49 & 4,90 & 4,37 & 6,31 \\
\hline & Déperdition d & $4,48 \%$ & & & & & & & & & & & & \\
\hline \multirow{3}{*}{ PEHD } & Iode (ppm) & 140,85 & 135,34 & 136,83 & 140,36 & 137,01 & 136,13 & 137,89 & 138,95 & 138,25 & 137,01 & 136,66 & 136,31 & 134,37 \\
\hline & $\Delta$ iode $(\mathrm{ppm})$ & 0,00 & 5,51 & 4,02 & 0,49 & 3,84 & 4,72 & 2,96 & 1,90 & 2,60 & 3,84 & 4,19 & 4,54 & 6,48 \\
\hline & Déperdition d & $4,60 \%$ & & & & & & & & & & & & \\
\hline \multirow{3}{*}{ PP } & Iode (ppm) & 140,85 & 137,98 & 136,66 & 136,13 & 136,48 & 136,13 & 136,66 & 137,54 & 137,01 & 135,78 & 137,01 & 136,13 & 134,54 \\
\hline & $\Delta$ iode $(\mathrm{ppm})$ & 0,00 & 2,87 & 4,19 & 4,72 & 4,37 & 4,72 & 4,19 & 3,31 & 3,84 & 5,07 & 3,84 & 4,72 & 6,31 \\
\hline & Déperdition d & $4,48 \%$ & & & & & & & & & & & & \\
\hline
\end{tabular}


Tableau 7 : Suivi du taux d'iode en fonction de l'emballage au Laboratoire.

\begin{tabular}{|c|c|c|c|c|c|c|c|c|c|c|c|c|c|c|}
\hline \multicolumn{2}{|c|}{$\begin{array}{c}\text { Semaines/ } \\
\text { Emballages }\end{array}$} & 0 & 1 & 2 & 3 & 4 & 5 & 6 & 7 & 8 & 9 & 10 & 11 & 12 \\
\hline \multirow{3}{*}{ 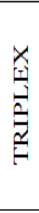 } & $\begin{array}{l}\text { Iode } \\
\text { (ppm) }\end{array}$ & 140,85 & 140,71 & 138,77 & 135,42 & 139,48 & 139,66 & 136,83 & 137,54 & 137,01 & 137,89 & 135,95 & 136,66 & 135,42 \\
\hline & $\begin{array}{c}\Delta \\
\text { iode } \\
\text { (ppm) }\end{array}$ & 0,00 & 0,14 & 2,08 & 5,43 & 1,37 & 1,19 & 4,02 & 3,31 & 3,84 & 2,96 & 4,90 & 4,19 & 5,43 \\
\hline & \multicolumn{14}{|c|}{ Déperdition d'iode: $3,85 \%$} \\
\hline \multirow{3}{*}{$\begin{array}{l}x \\
\hat{a} \\
\hat{5} \\
\hat{0}\end{array}$} & $\begin{array}{l}\text { Iode } \\
\text { (ppm) }\end{array}$ & 140,85 & 140,63 & 140,27 & 138,77 & 136,31 & 140,71 & 136,31 & 140,71 & 136,66 & 137,19 & 137,19 & 136,31 & 134,72 \\
\hline & $\begin{array}{c}\Delta \\
\text { iode } \\
\text { (ppm) }\end{array}$ & 0,00 & 0,22 & 0,58 & 2,08 & 4,54 & 0,14 & 4,54 & 0,14 & 4,19 & 3,66 & 3,66 & 4,54 & 6,13 \\
\hline & \multicolumn{14}{|c|}{ Déperdition d'iode: $4,35 \%$} \\
\hline \multirow{3}{*}{$\frac{\theta}{2}$} & $\begin{array}{l}\text { Iode } \\
(\mathrm{ppm})\end{array}$ & 140,85 & 135,34 & 138,60 & 139,66 & 137,19 & 135,78 & 136,13 & 137,01 & 137,36 & 136,83 & 136,48 & 136,66 & 135,25 \\
\hline & $\begin{array}{c}\Delta \\
\text { iode } \\
\text { (ppm) }\end{array}$ & 0,00 & 5,51 & 2,25 & 1,19 & 3,66 & 5,07 & 4,72 & 3,84 & 3,49 & 4,02 & 4,37 & 4,19 & 5,60 \\
\hline & \multicolumn{14}{|c|}{ Déperdition d'iode: $3,98 \%$} \\
\hline \multirow{3}{*}{$\hat{a}$} & $\begin{array}{l}\text { Iode } \\
(\mathrm{ppm})\end{array}$ & 140,85 & 135,86 & 138,25 & 139,30 & 138,07 & 137,72 & 137,01 & 137,54 & 137,54 & 137,72 & 136,13 & 136,13 & 134,01 \\
\hline & $\begin{array}{c}\Delta \\
\text { iode } \\
(\mathrm{ppm})\end{array}$ & 0,00 & 4,99 & 2,60 & 1,55 & 2,78 & 3,13 & 3,84 & 3,31 & 3,31 & 3,13 & 4,72 & 4,72 & 6,84 \\
\hline & \multicolumn{14}{|c|}{ Déperdition d'iode: 4,85\% } \\
\hline
\end{tabular}

Tableau 8 : Suivi du taux d'iode en fonction de l'emballage au Marché-Matam.

\begin{tabular}{llcccc}
\hline Mois/Emballages & & $\mathbf{0}$ & $\mathbf{1}$ & $\mathbf{2}$ & $\mathbf{3}$ \\
\hline \multirow{3}{*}{ TRIPLEX } & Iode $(\mathrm{ppm})$ & 140,85 & 138,42 & 134,54 & 135,25 \\
& $\Delta$ iode $(\mathrm{ppm})$ & 0,00 & 2,43 & 6,31 & 5,60 \\
& Déperdition d'iode: $3,98 \%$ & & & \\
\hline \multirow{3}{*}{ DUPLEX } & Iode $(\mathrm{ppm})$ & 140,85 & 135,42 & 134,54 & 134,19 \\
& $\Delta$ iode $(\mathrm{ppm})$ & 0,00 & 5,43 & 6,31 & 6,66 \\
& Déperdition d'iode: $4,73 \%$ & & & \\
\hline \multirow{3}{*}{ PEHD } & Iode (ppm) & 140,85 & 134,54 & 134,19 & 134,37 \\
& $\Delta$ iode (ppm) & 0,00 & 6,31 & 6,66 & 6,48 \\
& Déperdition d'iode: $4,60 \%$ & & & \\
PP & Iode (ppm) & 140,85 & 136,83 & 133,84 & 134,37 \\
& $\Delta$ iode (ppm) & 0,00 & 4,02 & 7,01 & 6,48 \\
& Déperdition d'iode: $4,60 \%$ & & & \\
\hline
\end{tabular}




\section{DISCUSSION}

Selon Donald. (2006), le sel est un moyen efficace de distribuer de l'iode à la population car il ne périme pas et il est consommé à des taux à peu près estimables. Le Sénégal est un pays de forte production de sel et se trouve au rang des grands producteurs en Afrique de l'Ouest. Cette production se fait au Nord (Gandiol), au Centre (Fatick et Kaolack) et au Sud (Kolda) du pays. D'après la caractérisation des différents sels échantillonnés dans différents sites d'extraction de sel comme mentionné dans le Tableau 4, le sel produit au Sénégal répond aux normes de qualité de sel alimentaire. D'autre part, la technique d'extraction explique les caractéristiques du sel produit: c'est le cas de Gandiol où l'exploitation est saisonnière; l'évaporation liée à l'ensoleillement, le grattage et le stockage facilitent la contamination du sel par les matières organiques (vase et poussière). En effet, ce sel est extrait au moment où la cristallisation est inachevée, entraînant un mélange de sel de calcium, de potasse ou de magnésie et d'impuretés. Ainsi, l'étude de la stabilité de l'iode par rapport à la nature du sel et l'emballage utilisé a été menée pour mieux appréhender les sources de déperdition d'iode. Aussi, la déperdition importante d'iode par rapport au sel fin pourrait être liée soit à la granulométrie soit à la présence élevée de matières insolubles. En comparant d'une part le sel fin et le sel criblé, nous pouvons dire que cette diminution est liée à la granulométrie des cristaux du sel. En effet, tous les autres paramètres pouvant être facteurs de déperdition d'iode sont plus importants dans le sel fin que dans le seul criblé. La granulométrie reste donc la seule cause de cette déperdition d'iode. Des études (Taga, 2005) qui ont confirmé la dégradation rapide du sel à gros grain dans le temps ont montré que les pourcentages de perte en iode vont de $9,98 \%$ pour les grains fins à $42,86 \%$ pour les gros. Bhat et al. (2008) ont rapporté une teneur moyenne en iode des sels en poudre (20,9 ppm) statistiquement plus élevée que celle des sels cristallins $(4,75 \mathrm{ppm})$ dans la région de Jammu en Inde. Quant à l'étude de Djonga et al. (2012), ils ont trouvé que le sel ayant les plus gros grains avait un taux d'iode nul. D'autre part, en comparant le sel fin et le sel tout venant, il devient aisé de corréler la perte en iode à la présence importante de matières insolubles. Ceci semble être évident, car le sel tout venant, n'ayant subi aucun traitement préalable avant iodation, est censé contenir beaucoup d'impuretés. A cet effet, nous en concluons que la déperdition d'iode dans le sel est liée à la granulométrie des cristaux et à la présence importante de matières insolubles. D'autres études menées au Tchad (Tidjani, 2000) et au Bénin (Gomina et al., 2011) ont montré que l'exposition à l'air libre facilite la déperdition de l'iode.

Que serait devenu l'iode contenu dans le sel au bout de six à douze mois de stockage ? Les travaux ayant été déroulés sur une période de trois mois, il semble difficile de le prédire. Cependant, l'allure des courbes donnant la variation des taux de diminution $(\Delta$ iode) au cours des 3 mois de suivi (Figures 7 , 8 et 9) permet de déduire qu'au bout de deux mois de stockage, la teneur en iode resterait constante. Cette observation nous autorise une extrapolation sur un an au minimum: nous y trouverons les valeurs mesurées au bout de trois mois d'expérience. Cependant, Tidjani (2000) indique que la moyenne de la teneur en iode dans le sel recueilli chez les grossistes du marché de Diguel est d'environ $20 \mathrm{ppm}$, largement inférieur aux normes requises par l'OMS pour les sels à la sortie des usines (Aquaron et Aquaron, 1991). Les grosses pertes en iode seraient dues en partie au mode de conservation et aux temps mis pour vendre ces sels après leur achat de l'usine. D'autres études en Ethiopie (Shawel et al., 2010) indiquent que le taux d'iode varie entre le producteur et le consommateur. 
Aussi, l'aspect emballage a-t-il été introduit pour voir l'effet de la lumière sur l'agent iodant (iodate de potassium $\mathrm{KIO}_{3}$ ). Parmi les emballages utilisés, le polypropylène (PP) à une opacité de 0 (donc transparent) a donné la même déperdition que les autres comme TRIPLEX (opacité de 2,4). Ainsi, cette diminution de l'iode dans le sel n'est pas due à la lumière. Par conséquent, cette dernière n'a pas d'effet sur l'iodate de potassium. D'une manière générale, l'iode est resté stable dans le sel quel que soit le type d'emballage utilisé à savoir le TRIPLEX, le DUPLEX, le PEHD et le PP. Ainsi, les emballages utilisés pour le sel qui sont le PEHD et le PP sont bien appropriés et ne sont pas à l'origine de déperditions significatives d'iode si celui-ci est du $\mathrm{KIO}_{3}$. Par rapport aux emballages, Taga et al. (2004) au Cameroun avaient indiqué que la bouteille en verre conserve mieux le sel iodé que le plastique ; ce dernier conserve encore mieux que le sac standard en polyéthylène. Gomina et al. (2011) confirme également dans leur étude que pour la plupart des sels de cuisine entreposés dans les flacons en verre, la teneur moyenne en iode était plus élevée que celle des sels en sachet et en plastique. Enfin, Djonga et al. (2012) affirme également que le meilleur récipient est celui fait en verre avec couvercle adapté.

\section{Conclusion}

Au terme de l'étude de la stabilité de l'iode par rapport à la nature du sel, il ressort que la déperdition d'iode par rapport à la teneur initiale est plus importante pour le sel criblé et le sel tout venant comparé au sel fin. Cela peut s'expliquer par plusieurs raisons tels que les caractéristiques de ces sels, la teneur en eau, les impuretés et les matières organiques du sel criblé et du sel tout venant. Aussi, pendant les trois mois de suivi dans les différents endroits de stockage, l'iode est resté stable dans le sel. Aucun impact de l'emballage utilisé sur la déperdition n'a été constaté. Des études plus poussées notamment sur des longues périodes de stockage seraient nécessaires pour approfondir ce travail.

\section{CONFLIT D'INTERETS}

Les auteurs déclarent qu'ils n'ont aucun conflit d'intérêts.

\section{REFERENCES}

Anderson M, De Benoist B, Rogers L. 2010. Epidemiology of iodine deficiency: salt iodisation and iodine status. Best Pract. Res. Clin. Endocrinol. Metab., 24(1): 111.

Allen L, de Benoist B, Dary O, Hurrell R. 2011. Directives sur l'enrichissement des aliments en micronutriments. Ed. Lindsay Allen : London ; 134.

Aquraon R, Aquaron C. 1998. Sels naturesl, sels iodés, sels fluorés. Actualités en diététique. 59-69 pp.

Association of Official Analytical Chemists (AOAC). 1974. Official Method of analysis $\left(15^{\text {th }} \mathrm{edn}\right)$. William Worwitz: Washington (USA), 1094.

de Benoist B, Delange F. 2002. La carence iodée : bilan et perspectives pour le futur. John LibbeyEurotext, 12(1): 917.

Cassandra Willyard. 2008. Salt of the Earth: The Public Health Community Employs a Mineral to Fight Infectious Disease. Geotimes, American Geological Institute.

Djonga O, Ali Mahamat M, Bessane C, Ache Danama K, Brahim Boy O. 2012. Comportements Alimentaires et Carence en Iode. Mali Medical, 27 : 13.

Donald G, Jr McNeil. 2006. In Raising the World's I.Q., the Secret's in the Salt, New York Times, 16 December 2006.

Gomina Assoumanou M, Zohoncon TM, Akpona SA. 2011. Evaluation de la teneur en iode des sels de cuisine dans 
les ménages de deux zones d'endémie goitreuse du Bénin. Int. J. Biol. Chem. Sci., 5(4): 1515-1526.

Lafoix C. 2005. Protocole d'aide à la décision de programme de fluoration du sel. Etude réalisée du 14 Avril 2005 au 13 Juillet 2005. 62 p.

Lagnane O. 2012. Production de sel iodé. Dasp - creneaux porteurs du secteur primaire- production de sel iode, $20 \mathrm{p}$.

Ministère de la Santé (Maroc). 2001. Les carences en micronutriments / ampleur du problème et stratégies de lutte, avril 2001, Rabat.

Ministère de la Santé (Maroc). 2008. Lutte contre les Troubles dus aux Carences en Micronutriments. Manuel à l'usage des professionnels de santé.

OMS. 1998. Rapport du Secrétariat soumis au Conseil exécutif pour information : Carence en iode. Conseil exécutif. $103^{\text {ème }}$ Session, 4 p.

Organisation des Nations Unies pour l'Alimentation et l'Agriculture (FAO). 2015. Carences en micronutriments.

Phyllis A. Lyday. 2005. Iodine and Iodine Compounds. In Ullmann's Encyclopedia of Industrial Chemistry. Wiley-VCH: Weinheim.

RODIER J. 1984. L'Analyse de l'Eau : Eaux Naturelles, Eaux Résiduaires, Eau de $\operatorname{Mer}\left(7^{\mathrm{ème}}\right.$ édn). Dunod : Paris ; 1365.

RODIER J. 1996. L'Analyse de l'Eau : Eaux Naturelles, Eaux Résiduaires, Eau de $\operatorname{Mer}\left(8^{\text {ème }}\right.$ édn). Dunod : Paris ; 1383.

Shawel D, Hagos S, Lachat CK, Kimanya E, Kolsteren P. 2010. Post-production losses in iodine concentration of salt hamper the control of iodine deficiency disorders: A case study in Northern Ethiopia. J. Health Popul. Nutr., 28(3): 238-44.

Taga I, Massouke Massouke DD, Ndomou M, Ngogang Yonkeu J. 2004. Problématique du sel iodé consommé au Cameroun. Cahiers d'Etudes et de Recherche Francophones/Santé, 14(3): 161-165.

Taga I. 2005. Troubles dus à la carence en iode au Cameroun : Etats des lieux et approches de solution : Aspects cliniques, biologiques et thérapeutiques. Thèse de Doctorat (PhD) Université de Yaoundé I, Cameroun, 116p.

Withney E, Rady Rolfes S. 2008. Understanding Nutrition (11 $1^{\mathrm{ème}}$ edn). Thomson Learning.

Pennington J, Douglass J, Bowes, Church's. 2005. Food Values of Portions Commonly Used, 18 E. Lippincott Williams and Wilkins. http://www. salines.com/sel-savoir-faire/ alimentation/sels-iodes-fluores/sel-iode Tidjani A. 2000. Teneur en iode des sels de cuisine vendus et consommés à N'Djamena. Mémoire de Maîtrise, Université de N'Djamena, 28 p.

Yomadji-Outengar O. 2002) S'Informer pour Mieux s'Alimenter. Les éditions du CEFOD : Tchad ; 60. 